\title{
Research of the Methylation Status of miR-124a Gene Promoter among Rheumatoid Arthritis Patients
}

\author{
Qiao Zhou, ${ }^{1}$ Li Long, ${ }^{1}$ Guixiu Shi, ${ }^{2}$ Jing Zhang, ${ }^{1}$ Tong Wu, ${ }^{1}$ and Bin Zhou ${ }^{1}$ \\ ${ }^{1}$ Department of Rheumatology, Sichuan Academy of Medical Science \& Sichuan Provincial People’s Hospital, No. 32 West Section 2, \\ Yihuan Road, Chengdu, Sichuan 610072, China \\ ${ }^{2}$ Department of Rheumatology, The First Affiliated Hospital of Xiamen University, Xiamen, Fujian 361003, China
}

Correspondence should be addressed to Bin Zhou; bingb2917@126.com

Received 1 May 2013; Accepted 5 September 2013

Academic Editor: Jianying Zhang

Copyright (C) 2013 Qiao Zhou et al. This is an open access article distributed under the Creative Commons Attribution License, which permits unrestricted use, distribution, and reproduction in any medium, provided the original work is properly cited.

\begin{abstract}
Objective. To analyze the methylation status of miR-124a loci in synovial tissues of rheumatoid arthritis (RA) patients using methylation-specific polymerase chain reaction (MSP). Materials and Methods. DNA obtained from the frozen tissue of 7 RA samples, 6 osteoarthritis (OA) samples, and 3 healthy controls were undergoing bisulfite conversion and then analyzed for miR124a promoter methylation using MSP assay. Results. miR-124-al and miR-124-a2 promoter methylation were both seen in $71.4 \%$ of RA samples compared to $16.7 \%$ of OA samples. miR-124-a3 promoter methylation was seen in $57.1 \%$ of RA samples and $0 \%$ of OA samples. All the three loci were unmethylated in 3 healthy controls. Conclusion. The methylation status of miR-124a seen in this study concurs with that reported in tumor cells, indicating epigenetic dysregulation constituents, a mechanism in the development of rheumatoid arthritis.
\end{abstract}

\section{Introduction}

Rheumatoid arthritis (RA) is a chronic, inflammatory, symmetrical polyarticular autoimmune disease affecting $\sim 1 \%$ of the population worldwide $[1,2]$. The main characteristic features of RA are persistent inflammation, synovium hyperplasia, lymphocyte infiltration, and abnormal proliferation of fibroblast-like synoviocytes (FLS), which eventually lead to progressive cartilage erosion and bone destruction [3]. Although the pathogenesis of RA is not clear, much evidence demonstrates that microRNAs (miRNAs) display important roles in immune response $[4,5]$. miRNAs are endogenous, short (about 19-25 nucleotides long) singlestranded, and noncoding RNAs that can influence the target mRNA processing at the posttranscriptional level [6] and play important roles in cell processes such as proliferation, apoptosis, differentiation, or even in tumorigenesis [7]. One of these important miRNAs is miR-124a. Accumulating evidence shows that miR-124a is downregulated in synovial tissues of RA patients compared with that of osteoarthritis (OA) patients [8]. For example, Nakamachi et al. found that its level in RA FLS was less than one-sixth of that seen in OA
FLS [9]. However, the mechanism of the aberrant expression of miR-124a in RA synovial tissues is still unknown. It is found that miR-124a has three genomic loci (miR-124a-1 (8p23.1), miR-124a-2 (8q12.3), and miR-124a-3 (20q13.33)) that encode for the same mature miRNA. Interestingly, the miR-124a1 and miR-124a-3 genes are located within CpG islands, whereas miR-124a-2 is 760 bp downstream of a CpG island. Studies in several cancer cells demonstrated that all the three loci are silenced by the hypermethylation of its promoter $[10,11]$. Agirre et al. pointed out that the corresponding CpG islands of miR-124a-1 and miR-124a-3 are frequently methylated in acute myeloid leukemia [12]. Since reported data showed that the expression of miR-124a was suppressed in RA synovial tissues, it would be of interest to investigate whether epigenetic mechanism, especially DNA methylation, has played a role in it.

\section{Materials and Methods}

2.1. Synovial Samples. Synovial tissues were obtained from RA patients, OA patients, and joint trauma patients (healthy control specimens) undergoing total knee arthroplasty from 
TABLE 1: Clinical characteristics of the patients*

\begin{tabular}{|c|c|c|c|c|c|c|c|}
\hline Patient & Age & Sex & Disease duration (years) & Presurgical CRP (mg/dL) & Presurgical ESR (mm/h) & DAS 28 & Medications \\
\hline RA1 & 72 & $\mathrm{~F}$ & 17 & 1.9 & 17 & 2.42 & $\begin{array}{l}\text { MTX } 10 \text { mg qw } \\
\text { LEF } 20 \text { mg qd }\end{array}$ \\
\hline RA2 & 58 & $\mathrm{~F}$ & 9 & 1.5 & 21 & 2.16 & $\begin{array}{l}\text { MTX } 12.5 \text { mg qw } \\
\text { LEF } 10 \mathrm{mg} \text { qd }\end{array}$ \\
\hline RA3 & 63 & $\mathrm{M}$ & 21 & 2.7 & 13 & 1.87 & $\begin{array}{l}\text { Pred. } 7.5 \mathrm{mg} \mathrm{qd} \\
\text { MTX } 12.5 \mathrm{mg} \text { qw }\end{array}$ \\
\hline RA4 & 65 & $\mathrm{~F}$ & 15 & $<1$ & 2 & 1.21 & $\begin{array}{l}\text { Pred. } 5 \mathrm{mg} \text { qd } \\
\text { LEF } 20 \mathrm{mg} \text { qd }\end{array}$ \\
\hline RA5 & 59 & $\mathrm{M}$ & 6 & 3.2 & 5 & 1.49 & $\begin{array}{l}\text { Pred. } 5 \mathrm{mg} \text { qod } \\
\text { MTX } 10 \mathrm{mg} \mathrm{qw}\end{array}$ \\
\hline RA6 & 66 & $\mathrm{~F}$ & 8 & $<1$ & 9 & 1.23 & LEF $20 \mathrm{mg}$ qd \\
\hline RA7 & 61 & $\mathrm{~F}$ & 13 & 5.3 & 28 & 2.62 & MTX 15 mg qw \\
\hline OA1 & 72 & $\mathrm{~F}$ & - & $<1$ & 3 & - & - \\
\hline $\mathrm{OA} 2$ & 75 & $\mathrm{M}$ & - & $<1$ & 2 & - & - \\
\hline OA3 & 69 & $\mathrm{M}$ & - & $<1$ & 4 & - & - \\
\hline OA4 & 74 & $\mathrm{M}$ & - & 3.3 & 9 & - & - \\
\hline OA5 & 68 & $\mathrm{~F}$ & - & $<1$ & 3 & - & - \\
\hline OA6 & 70 & $\mathrm{M}$ & - & 2.8 & 11 & - & - \\
\hline $\mathrm{C} 1$ & 39 & $\mathrm{M}$ & - & & & & \\
\hline $\mathrm{C} 2$ & 45 & $\mathrm{M}$ & - & & & & \\
\hline C3 & 43 & $\mathrm{~F}$ & - & & & & \\
\hline
\end{tabular}

* RA: rheumatoid arthritis; OA: osteoarthritis; CRP: C-reactive protein; ESR: erythrocyte sedimentation rate; DAS: disease activity score; MTX: methotrexate; Pred.: prednisone; LEF: leflunomides; C: control.

October 2012 to April 2013 in Sichuan Provincial People's Hospital. Tissue was snap-frozen and stored at $-80^{\circ} \mathrm{C}$. RA and $\mathrm{OA}$ were diagnosed according to the criteria of the American College of Rheumatology $[13,14]$. The clinical characteristics of the patients are shown in Table 1. Samples were obtained in accordance with the Declaration of Helsinki Ethical Principles for Medical Research Involving Human Subjects, as approved by the World Medical Association. All patients signed informed consent forms, and the study was approved by the Ethics Committees of Sichuan Provincial People's Hospital.

2.2. DNA Extraction and Bisulfite Conversion. Genome DNA was extracted from $25 \mathrm{mg}$ of frozen synovial tissue using the PureLink Genomic DNA mini kit (Invitrogen, USA). DNA was quantitated using the Nanodrop (Nanodrop technologies, USA). $2 \mu \mathrm{g}$ DNA was used for bisulfite conversion as described [15]. Modified DNA was purified using the QIAEX II Gel Extraction kit (QIAGEN, Germany) according to the manufacturer.

2.3. Methylation-Specific PCR. The DNA methylation status was analyzed by methylation-specific PCR (MSP) using primers specific for either the methylated or bisulfate modified unmethylated DNA. $1.5 \mu \mathrm{L}$ bisulfite-converted DNA was amplified using $0.15 \mu \mathrm{L}$ primers (Sangon Biotech, Shanghai), $0.45 \mu \mathrm{L} \mathrm{MgCl}_{2}$ (25 mM), $1.8 \mu \mathrm{L}$ dNTP (2.5 mM each), $6.75 \mu \mathrm{L}$ 2x GC buffer (I) (Takara, Japan), and $1.6 \mu \mathrm{L}$ Takara LA taq polymerase (Takara, Japan). Each step included methylated (RKO, ATCC CRL-2577) and unmethylated (MGC803, ATCC) controls along with nontemplate control. The amplified products were run on a $2 \%$ agarose gel with an expected size of 164,185 , and $150 \mathrm{bp}$ (three loci each) for a methylated product and 165, 180, and $155 \mathrm{bp}$ (three loci each) for an unmethylated product.

2.4. Statistical Analysis. Statistical comparisons between groups were carried out by Chi square test or Fisher's exact test. $P$ values less than 0.05 were considered significant.

\section{Results}

3.1. General Clinical Features. The mean age of the 7 RA patients was 63 (range 58-72) years, with a female to male ratio of $2.5: 1$. The mean presurgical CRP and ESR were $2.4 \pm 1.5 \mathrm{mg} / \mathrm{dL}$ and $14 \pm 9 \mathrm{~mm} / \mathrm{h}$, respectively. The mean DAS28 was $1.86 \pm 0.57$. The three healthy control patients underwent surgery because of car accident, falling off, and fighting, respectively.

3.2. Methylation Status of miR-124a. The methylation status of miR-124a was evaluated in $7 \mathrm{RA}, 6 \mathrm{OA}$, and 3 control synovial tissues (Figure 1). All of the RA tissues were hypermethylated: 5 for miR-124a-1 (5/7), 5 for miR-124a-2 (5/7), and 4 for miR-124a-3 (4/7). Among them, 4 tissues (4/7) showed promoter methylation of all three gene loci, and 

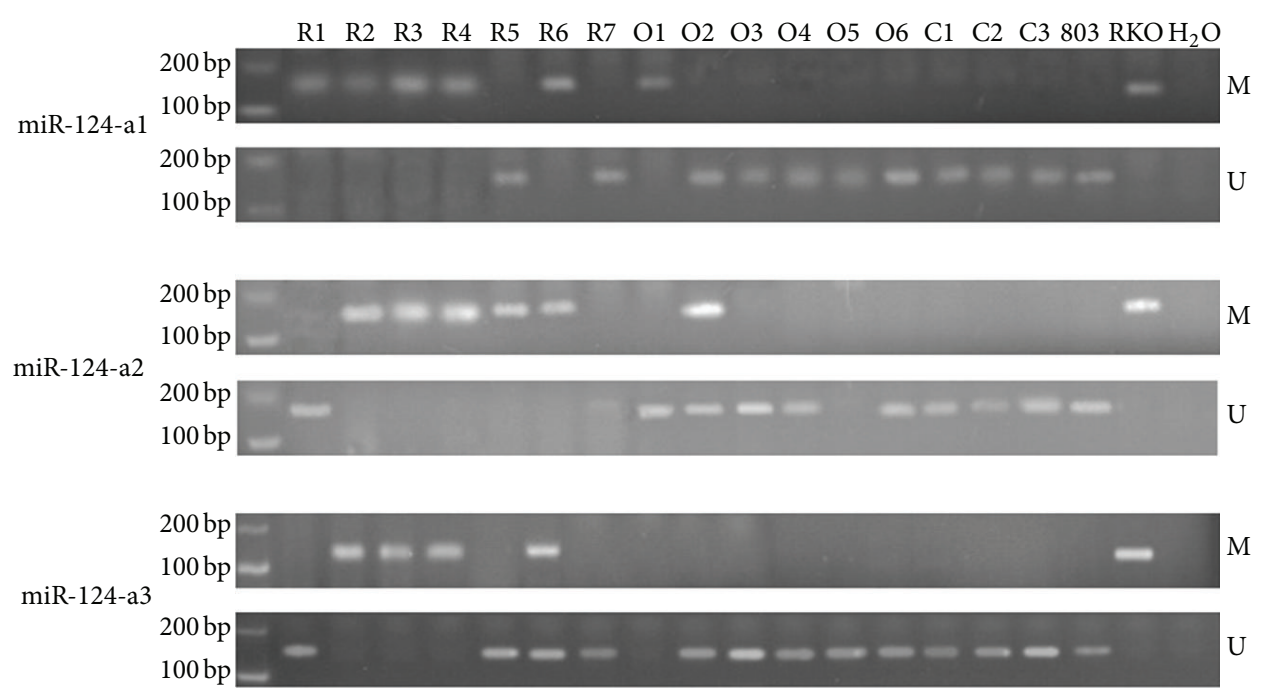

FIGURE 1: Gel electrophoresis of the MSP products for miR-124-a1, miR-124-a2, and miR-124-a3 in RA, OA patients, and healthy controls. R: rheumatoid arthritis; O: osteoarthritis; C: healthy control; 803 is unmethylated positive control; $\mathrm{RKO}$ is methylated positive control; $\mathrm{H}_{2} \mathrm{O}$ is blank control.

TABLE 2: Methylation status of miR-124a in synovial tissues of RA and $\mathrm{OA}^{*}$.

\begin{tabular}{lccccc}
\hline \multirow{2}{*}{ Gene } & \multicolumn{3}{c}{ Methylation frequency } & \multicolumn{2}{c}{$P$ value } \\
& RA & OA & C & RA versus OA RA versus C \\
\hline miR-124-a1 & $71.4 \%$ & $16.7 \%$ & 0 & 0.07 & 0.08 \\
miR-124-a2 & $71.4 \%$ & $16.7 \%$ & 0 & 0.07 & 0.08 \\
miR-124-a3 & $57.1 \%$ & 0 & 0 & 0.05 & 0.17 \\
All three loci & $57.1 \%$ & 0 & 0 & 0.05 & 0.17 \\
\hline
\end{tabular}

${ }^{*}$ RA: rheumatoid arthritis; OA: osteoarthritis; C: healthy control.

the frequency was $57.1 \%$, higher than that of OA $(0,0 / 6)$ and control $(0,0 / 3)$ (Table 2). The methylation frequency of the three genes in RA synovial tissues has no statistical significance compared to each other.

\section{Discussion}

Rheumatoid arthritis is one of the common autoimmune diseases, and the molecular mechanism of its pathogenesis is unclear now. miRNAs have been implicated in the pathogenesis of malignant and nonmalignant disease. The microRNA miR-124a was initially identified as a crucial regulator involved in neurogenesis [16]. In neuronal tissues, miR124a contributes to the differentiation of neural progenitors into mature neurons [17]. Pierson et al. reported that the $3^{\prime}$ UTR of CDK- 6 mRNA is a direct target of miR-124 and that CDK-6 expression is suppressed by miR-124 overexpression in medulloblastoma cell lines [18]. Nakamachi et al. confirmed that the expression of CDK-6 protein was higher in RA FLS than in OA FLS and that CDK-6 expression was suppressed when pre-miR-124a was transfected into RA FLS; thus, the cell cycle was arrested at the G1 phase [9]. Since CDK-6 is known as a G1/S phase regulator, it is speculated that miR-124a is an important regulator of the G1/S transition in synovial tissue as well as in tumors.

We decided to focus on the methylation status of miR124a in RA synovial tissues for several reasons. (a) The expression of miR-124a is downregulated in RA synovial tissues. (b) Hypermethylation of gene promoters is a frequent mechanism of gene silencing. (c) The three loci of miR124a are either located within a CpG island or somewhere downstream a CpG island. (d) It is reported that miR-124a has been silenced by gene promoter hypermethylation in several cancer cell lines. The results of our study support the approach, showing that the gene promoter of miR$124 \mathrm{a}$ is hypermethylated, and this might associate with its downregulation in RA synovial tissues.

The methylation frequencies of the three gene loci observed among RA synovial tissues (57.1\% 71.4\%) were much higher than that observed among OA $(0 \sim 16.7 \%)$ and control synovial tissues (0). However, the $P$ values were not so significant considering the small sample size.

We obtained good-quality DNA from all frozen tissues, yet there were some RA samples that showed both methylated and unmethylated band. We considered the following reasons: (a) although we carefully removed all connective tissue and fat, the area chosen might still have very little nonsynovial tissue to confound the results. It would be pertinent to point out that small amounts of contaminating nonsynovial tissues could become a source of contamination and could provide false negative results. This issue emphasizes the need to carefully choose samples for detection of methylation status, therefore avoiding potential confounders. (b) Regions within the synovial tissue might be variably methylated, even within the individual clones of the same tissue sample, and the synovial tissue could be a mosaic of variable degrees of methylation. Thus we speculate mosaicism could have contributed to our findings here. This issue could be solved by bisulfite-sequencing. 
This study highlights the methylation pattern of RA synovial tissues compared with that of OA and healthy control. Although it has some limitations such as the number of samples analyzed, the study still provides useful information on promoter methylation pattern of miR-124a. This epigenetic dysregulation of miR-124a in RA synovial tissue constitutes an emerging mechanism implicated in the development of RA and will provide us with new excellent targets for DNA demethylating agents.

\section{Conflict of Interests}

None of the authors had any conflict of interests.

\section{Acknowledgment}

This study was funded by the National Natural Science Foundation of China (81102272).

\section{References}

[1] A. Ruyssen-Witrand, A. Constantin, A. Cambon-Thomsen, and M. Thomsen, "New insights into the genetics of immune responses in rheumatoid arthritis," Tissue Antigens, vol. 80, no. 2, pp. 105-118, 2012.

[2] A. Gramling and J. R. O’Dell, "Initial management of rheumatoid arthritis," Rheumatic Diseases Clinics of North America, vol. 38, no. 2, pp. 311-325, 2012.

[3] F. A. H. Cooles and J. D. Isaacs, "Pathophysiology of rheumatoid arthritis," Current Opinion in Rheumatology, vol. 23, no. 3, pp. 233-240, 2011.

[4] S. P. Persengiev, "miRNAs at the crossroad between hematopoietic malignancies and autoimmune pathogenesis," Discovery Medicine, vol. 13, no. 70, pp. 211-221, 2012.

[5] M. de Santis and C. Selmi, "The therapeutic potential of epigenetics in autoimmune diseases," Clinical Reviews in Allergy \& Immunology, vol. 42, no. 1, pp. 92-101, 2012.

[6] C. L. Bartels and G. J. Tsongalis, "Mini-reviews micrornas: novel biomarkers for human cancer," Clinical Chemistry, vol. 55, no. 4, pp. 623-631, 2009.

[7] C. Z. Chen, "MicroRNAs as oncogenes and tumor suppressors," The New England Journal of Medicine, vol. 353, no. 17, pp. 17681771, 2005.

[8] C.-G. Miao and Y.-Y. Yang, "New advances of microRNAs in the pathogenesis of rheumatoid arthritis, with a focus on the crosstalk between DNA methylation and the microRNA machinery," Cellular Signalling, vol. 25, no. 5, pp. 1118-1125, 2013.

[9] Y. Nakamachi, S. Kawano, M. Takenokuchi et al., "MicroRNA$124 \mathrm{a}$ is a key regulator of proliferation and monocyte chemoattractant protein 1 secretion in fibroblast-like synoviocytes from patients with rheumatoid arthritis," Arthritis \& Rheumatism, vol. 60, no. 5, pp. 1294-1304, 2009.

[10] A. Lujambio, S. Ropero, E. Ballestar et al., "Genetic unmasking of an epigenetically silenced microRNA in human cancer cells," Cancer Research, vol. 67, no. 4, pp. 1424-1429, 2007.

[11] A. Lujambio and M. Esteller, "CpG island hypermethylation of tumor suppressor microRNAs in human cancer," Cell Cycle, vol. 6, no. 12, pp. 1455-1459, 2007.
[12] X. Agirre, A. Vilas-Zornoza, A. Jiménez-Velasco et al., "Epigenetic silencing of the tumor suppressor microRNA Hsa-miR124a regulates CDK6 expression and confers a poor prognosis in acute lymphoblastic leukemia," Cancer Research, vol. 69, no. 10, pp. 4443-4453, 2009.

[13] F. C. Arnett, S. M. Edworthy, D. A. Bloch et al., "The American Rheumatism Association 1987 revised criteria for the classification of rheumatoid arthritis," Arthritis \& Rheumatism, vol. 31, no. 3, pp. 315-324, 1988.

[14] R. Altman, E. Asch, D. Bloch et al., "Development of criteria for the classification and reporting of osteoarthritis: classification of osteoarthritis of the knee," Arthritis \& Rheumatism, vol. 29, no. 8, pp. 1039-1049, 1986.

[15] J. G. Herman, J. R. Graff, S. Myöhänen, B. D. Nelkin, and S. B. Baylin, "Methylation-specific PCR: a novel PCR assay for methylation status of CpG islands," Proceedings of the National Academy of Sciences of the United States of America, vol. 93, no. 18, pp. 9821-9826, 1996.

[16] W. P. Kloosterman and R. H. A. Plasterk, "The diverse functions of microRNAs in animal development and disease," Developmental Cell, vol. 11, no. 4, pp. 441-450, 2006.

[17] C. Conaco, S. Otto, J. J. Han, and G. Mandel, "Reciprocal actions of REST and a microRNA promote neuronal identity," Proceedings of the National Academy of Sciences of the United States of America, vol. 103, no. 7, pp. 2422-2427, 2006.

[18] J. Pierson, B. Hostager, R. Fan, and R. Vibhakar, "Regulation of cyclin dependent kinase 6 by microRNA 124 in medulloblastoma," Journal of Neuro-Oncology, vol. 90, no. 1, pp. 1-7, 2008. 


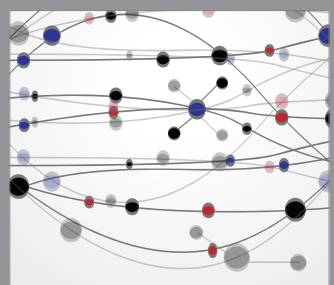

The Scientific World Journal
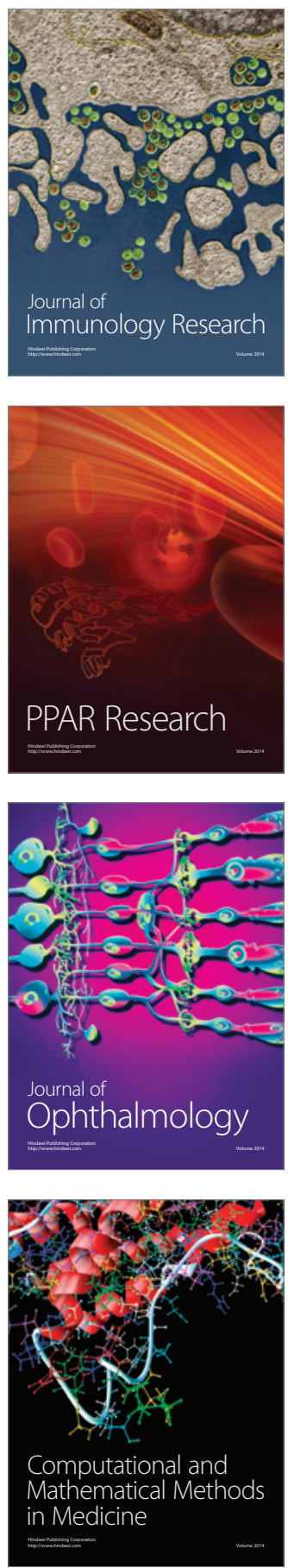

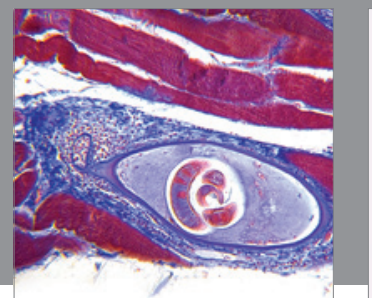

Gastroenterology

Research and Practice
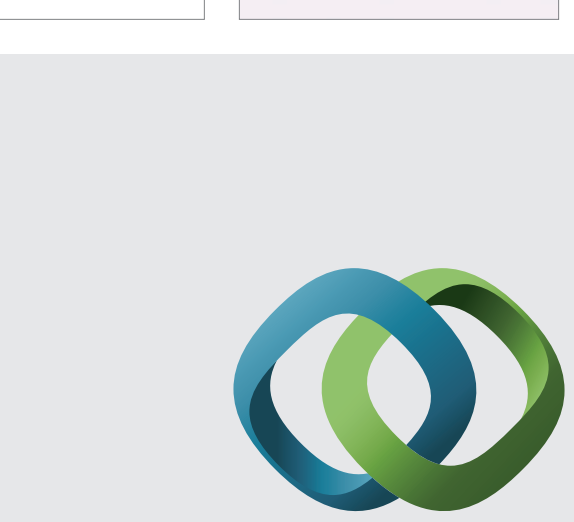

\section{Hindawi}

Submit your manuscripts at

http://www.hindawi.com
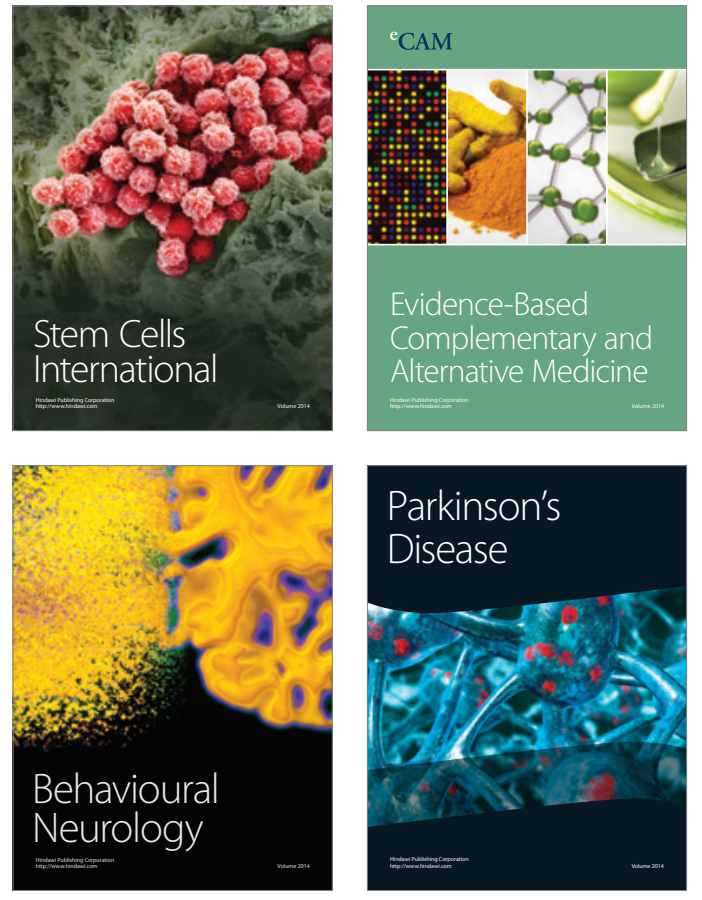
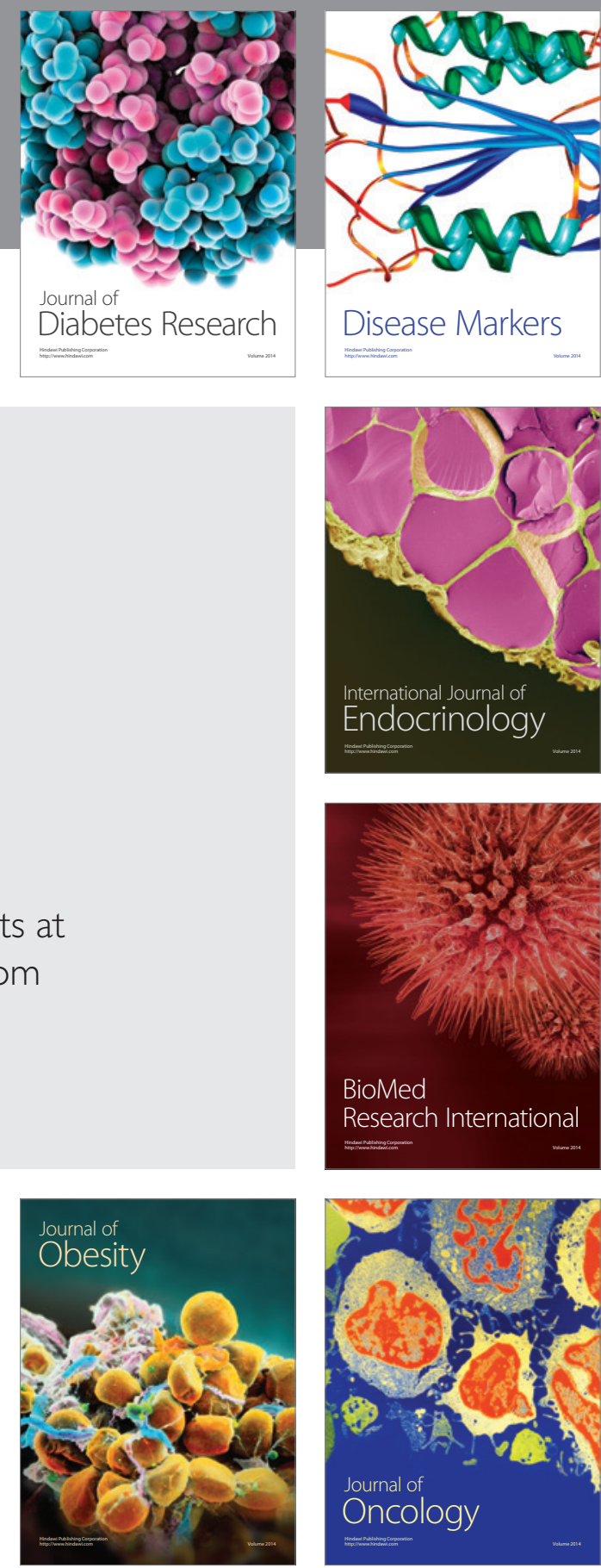

Disease Markers
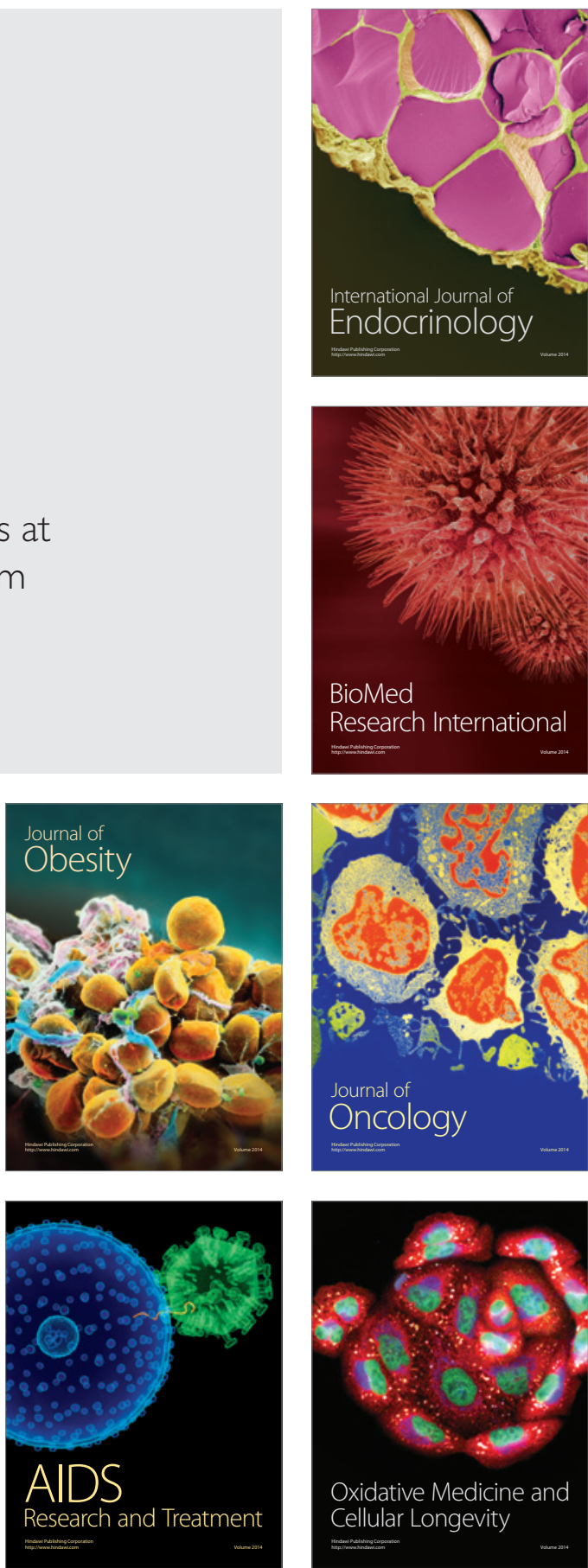